in from the outer part of the disk. Such pebbles had high $\mu^{48} \mathrm{Ca}$ values (about 200 p.p.m.), which are typical of the most primitive meteorites (carbonaceous chondrites) that are thought to have formed beyond Jupiter ${ }^{6}$. The mean $\mu^{48} \mathrm{Ca}$ value in the inner disk therefore increased progressively. As a consequence, bodies that continued to grow to the size of Vesta $\left(530 \mathrm{~km}\right.$ in diameter) reached $\mu^{48} \mathrm{Ca}$ values of -100 p.p.m., and those that grew to the size of Mars $(6,800 \mathrm{~km}$ in diameter) reached $\mu^{48} \mathrm{Ca}$ values of -20 p.p.m (Fig. 1).

Earth and the Moon have $\mu^{48} \mathrm{Ca}$ values of about 0 p.p.m. It is generally assumed that Earth formed after the disappearance of the protoplanetary disk, as a result of collisions of planetary embryos with masses similar to that of Mars; the body that collided with early Earth to give rise to the Moon was one of these embryos. However, this formation scenario is not possible if Schiller and colleagues' proposal is correct. Planetary embryos with the mass of Mars would have $\mu^{48} \mathrm{Ca}$ values of -20 p.p.m., and therefore an 'Earth' resulting from the merger of these bodies would have a similar composition.

Instead, for Earth to have a $\mu^{48} \mathrm{Ca}$ value of about 0 p.p.m., the embryos would need to have grown by pebble accretion to masses roughly half that of Earth. In turn, the Moonforming impactor would need to have had a mass comparable to that of the embryos to have the same $\mu^{48} \mathrm{Ca}$ value, supporting the theory that the Moon resulted from the collision of two bodies with half-Earth masses ${ }^{7}$.

A potential problem is that hafniumtungsten radioactive chronometry indicates that Earth reached $63 \%$ of its present mass only after a duration of between 11 million and 24 million years, depending on the type of core-mantle equilibration that occurred during the collision of the embryos ${ }^{8}$. It is therefore difficult to imagine that such embryos grew to bodies with half-Earth masses in the disk's putative lifetime of 5 million years ${ }^{9}$.

But perhaps the embryos did reach the size of one-third of Earth's mass (three times the mass of Mars). Such a proposal cannot be discounted as a compromise between the authors' correlation of calcium-isotope compositions with planetary masses and the chronological constraints on Earth's accretion. The fraction of Earth's mass that would have been accreted from the outer disk (estimated at about $40 \%$ in the authors' study) would be higher than calculated previously ${ }^{10,11}$, but such computations did not consider some of the building blocks of Earth to have ureilite-like compositions.

Schiller and colleagues' view of accretion in the Solar System is in sharp contrast with that presented by two previous studies ${ }^{6,12}$. These reports concluded that the flux of pebbles from the outer disk was shut down during the first million years of the disk's lifetime by the formation of Jupiter. This prevented bodies in the inner Solar System from accumulating large amounts of water ice, explaining why such bodies are mostly dry ${ }^{12}$, and maintained an isotopic dichotomy between two types of meteorite: ordinary and carbonaceous chondrites ${ }^{6}$.

The composition of ordinary chondrites is difficult to account for using Schiller and colleagues' model. Unlike large bodies, which should grow continuously by pebble accretion, the smaller parent bodies of chondrites should form suddenly, from clusters of pebbles that are generated by a mechanism known as a streaming instability ${ }^{1}$. In the case of ordinary chondrites, this would have happened in the inner Solar System at a late stage in its formation, after the time by which Mars had accreted most of its mass. Ordinary chondrites should therefore represent a snapshot of the composition of the late disk, and have a positive value of $\mu^{48} \mathrm{Ca}$ in the authors' model. But, in reality, such chondrites have $\mu^{48} \mathrm{Ca}$ values of -35 p.p.m.

Schiller et al. explain this discrepancy by speculating that pebble accretion and the streaming instability have differing preferences for pebble size. The pebbles that came from the outer disk were small and, although they were efficiently accreted by large bodies, they barely participated in the streaming instability. Consequently, ordinary chondrites formed mostly from larger pre-existing pebbles called chondrules, which typically have negative values of $\mu^{48} \mathrm{Ca}$. The validity of this proposal will need to be checked using high-resolution numerical simulations of the pebble-accretion and streaming-instability processes.

The authors' work adds a missing piece to the jigsaw puzzle of planet formation that will need to be connected with the other pieces provided by isotopic, chemical, chronological and dynamical constraints. Although the puzzle seems more complete than before, perhaps some other key pieces are still missing. $\square$

Alessandro Morbidelli is at the Observatoire de la Côte d'Azur, CS 34229, 06304 Nice

Cedex 4, France.

e-mail:morby@obs-nice.fr

1. Johansen, A., Mac Low, M.-M., Lacerda, P. \& Bizzarro, M. Sci. Adv. 1, e1500109 (2015).

2. Schiller, M., Bizzarro, M. \& Fernandes, V. A. Nature 555, 507-510 (2018)

3. Schiller, M., Baker, J. A. \& Bizzarro, M. Geochim. Cosmochim. Acta 74, 4844-4864 (2010).

4. Wilson, L., Goodrich, C. A. \& Van Orman, J. A Geochim. Cosmochim. Acta 72, 6154-6176 (2008).

5. Levison, H. F., Kretke, K. A. \& Duncan, M. J. Nature 524, 322-324 (2015)

6. Kruijer, T. S., Burkhardt, C., Budde, G. \& Kleine, T. Proc. Natl Acad. Sci. USA 114, 6712-6716 (2017).

7. Canup, R. M. Science 338, 1052-1055 (2012).

8. Kleine, T. et al. Geochim. Cosmochim. Acta 73, 5150-5188 (2009).

9. Mamajek, E. E. AIP Conf. Proc. 1158, 3-10 (2009)

10.Fitoussi, C., Bourdon, B. \& Wang, X. Earth Planet. Sci. Lett. 434, 151-160 (2016).

11.Dauphas, N. Nature 541, 521-524 (2017)

12. Morbidelli, A. et al. Icarus 267, 368-376 (2016)

\title{
A mixed model of neuronal diversity
}

\section{Two groups have sequenced RNA from thousands of single cells, making the deepest ventures yet into the origins of neuronal diversity in the neocortex of the developing mammalian brain. SEE ARTICLE P.457}

\section{LUDOVIC TELLEY \& DENIS JABAUDON}

$\mathrm{B}$ iologists have long been preoccupied with classifying diverse objects into meaningful categories. Even at the beginning of genetics, Gregor Mendel classified peas as smooth or wrinkled, to infer the mechanisms underlying the inheritance of specific features. Neurons, like peas, come in different shapes and types, but how many neuronal cell types exist and how neuronal diversity emerges during development is unclear. Answering these questions is important, because the diversity of neurons determines the diversity of circuits that can be built and, by extension, the scope of an animal's behavioural repertoire. Two groups (one writing in Nature ${ }^{1}$ and one in Science ${ }^{2}$ ) now investigate the origins of neuronal diversity in the developing neocortex, a brain region that is the source of mammals' complex behavioural and cognitive abilities ${ }^{3}$.

Two opposing - although not mutually exclusive - scenarios account for the generation of distinct kinds of neuron across the nervous system, and in the cerebral cortex in particular ${ }^{3-6}$. In the first scenario, diverse neuronal subtypes are born from correspondingly diverse progenitor cells (Fig. 1a). This is known as the premitotic model, because diversity arises in progenitors, before neurons are actually generated (neuronal birth occurs through mitotic cell division). In this 
scenario, the diversity of progenitors might result from the cells' locations in different parts of the brain, or from their generation at different times. In the second scenario, known as the postmitotic model, progenitors are homogeneous, and diversity arises in neurons as they develop, including through interactions with the surrounding environment, which sculpt these cells into more-refined adult cell types (Fig. 1b).

In the current studies, the groups investigated how diversity arises in the neocortex by using single-cell RNA sequencing to identify and define distinct cell types by their transcriptional signatures. In contrast to previous approaches, in which cells were lumped together to yield average transcriptional activities, this technology allows several thousand individual cells to be simultaneously singled out and sequenced, yielding a more finegrained view of neurons' molecular identities ${ }^{7}$.

In the first study, Mayer et al. ${ }^{1}$ (page 457) focused on a population of neurons in the mouse neocortex that releases the inhibitory neurotransmitter molecule GABA. These neurons arise from progenitors located in three transient structures of the developing mammalian brain, called ganglionic eminences ${ }^{8}$. Using single-cell RNA sequencing, the authors revealed that transcriptional programs are largely conserved in progenitors across the ganglionic eminences. Only a select set of genes is differentially expressed between the three regions - a limited level of premitotic diversity that is consistent with the postmitotic model.

Next, the researchers used elaborate bioinformatics approaches to further reconstruct the developmental trajectory of each neuronal subtype. This analysis demonstrated a link between the initial diversity of immature neurons and the diversity in mature neuronal subtypes, consistent with the premitotic model. The group thus showed that mature neuronal properties are already distinguishable in a rough form in newborn neurons. Subtypes then become more crisply defined as neurons, poised in the genetic ground state dictated by progenitors; they then differentiate and interact with the environment. Altogether, these data support a mixed model of development in which diversification occurs in both pre- and postmitotic cells (Fig. 1c).

In the second study, Nowakowski et al. focused on excitatory neurons that produce the neurotransmitter glutamate, in two neocortical

\section{a Premitotic model}

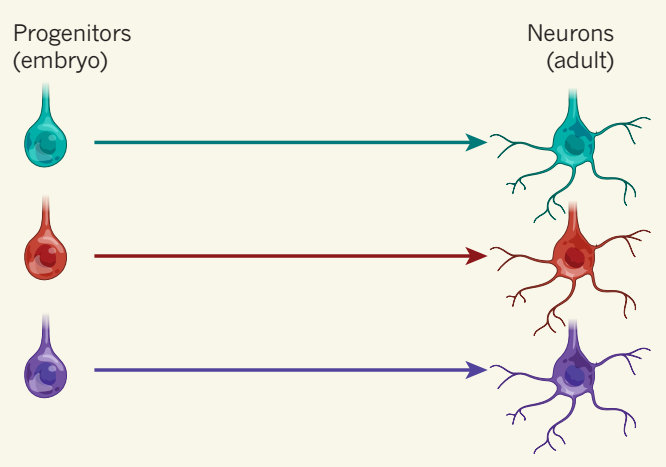

b Postmitotic model

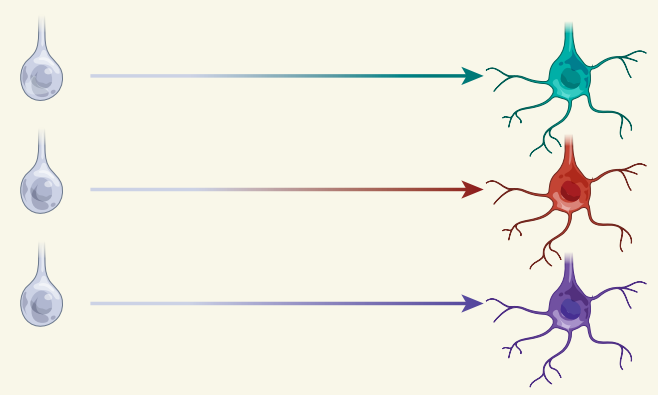

c Mixed model

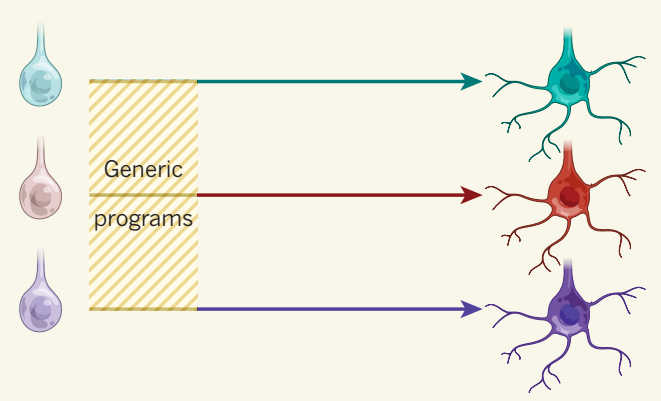

Figure 1 | Sources of neuronal diversity. a, In the 'premitotic' model, neuronal diversity arises in progenitor cells, owing to differences in either their location or the timing of their generation (different colours represent different gene-expression patterns). Diverse progenitors give birth to diverse neurons (the beginning of each arrow indicates neuron birth), which mature into diverse adult neurons.

b, In the 'postmitotic' model, diversity emerges only after progenitors have differentiated into neurons, owing in part to interactions with the environment. c, Two groups ${ }^{1,2}$ provide evidence that, in the neocortex of the mammalian brain, relatively few differences exist across progenitors (similar to the postmitotic model), but these differences are sufficient to drive neuronal diversity (as in the premitotic model), generating a mixed model. There seem to be generic transcriptional programs across neuronal types in the early stages of their development. (Modified from ref. 5.)

but explanations involving pre- or postmitotic divergences have both been put forward ${ }^{4,5}$.

The authors explored how these spatial differences emerge using similar experimental and analytical tools to those of Mayer and colleagues. Nowakowski et al. found that only select sets of genes are differentially expressed between progenitors across the two cortical regions, and that differences increase as neurons mature. These findings thus support a 'soft' premitotic situation that leaves leeway for extrinsic factors to drive the developmental trajectories of postmitotic newborn neurons (Fig. 1c). Despite fundamental differences in the biology of the cell types studied, then, the groups reach similar conclusions.

An intriguing finding made by Nowakowski et al. is that glutamateproducing neurons share their initial transcriptional trajectories with GABA-producing neurons. Similarly, Mayer et al. found that newborn neurons from all ganglionic eminences initially transit through overlapping transcriptional ground states. Thus, neuronal subtypes seem to emerge from subtype-specific processes that are superimposed on more-generic developmental programs.

These two studies shed longawaited light on the origin of neuronal subtypes. However, the quest to understand neuronal diversity is only just beginning. Mendel's classification of peas was later revisited by Raphael Weldon, who argued that there were many more categories than originally described ${ }^{9,10}$. Similarly, other studies might reveal more diversity than is reported in the current papers because, inevitably, the current studies are constrained by the criteria used to define cell classes.

To explain, single-cell RNA sequencing, in principle, provides an objective and comprehensive criterion for cellular classification. However, the granularity of the transcriptional assessment - factors such as sequencing quality and which kinds of RNA are analysed - is a key parameter in delineating cell types. Thus, future areas in human fetuses - the prefrontal cortex and the primary visual cortex, which are involved in behavioural planning and in vision, respectively. Neurons in these regions are organized into archetypal cortical columns, which are core functional units of circuits. The precise structure of these columns is thought to be tweaked across cortical areas to allow for different functions ${ }^{3-5}$. How these spatial differences emerge is poorly understood, studies might reveal more progenitor types than those found here, and tilt the balance towards a more premitotic view of diversity. In fact, one recently published study ${ }^{11}$ reported substantial diversity in immature neurons. Furthermore, sequencing approaches differentiate between cell types only on the basis of molecular differences; other criteria such as electrophysiological properties and anatomy are probably crucial, even at 
early developmental stages, for a complete definition of cell types.

How identity progresses with time in postmitotic neurons remains elusive. It will be particularly useful to dissect the extent to which external factors, including sensory signals from the peripheral nervous system, drive differentiation and further diversification of neurons within these genetically poised subtypes. Resolving these issues will be central not only to addressing the mechanisms that generate neuronal diversity, but also to characterizing and understanding inter-individual differences in circuit structure and function.

Finally, both studies identify disease-related genes among the cell-type-specific transcripts, including some associated with autism and schizophrenia. As such, they provide a valuable resource for those seeking to understand the mechanisms underlying these disorders.

Ludovic Telley is in the Department of Fundamental Neuroscience, University of Lausanne, 1005 Lausanne, Switzerland. Denis Jabaudon is in the Department of Basic Neurosciences, University of Geneva, 1211 Geneva, Switzerland. e-mails:ludovic.telley@unil.ch; denis.jabaudon@unige.ch
1. Mayer, C. et al. Nature 555, 457-462 (2018).

2. Nowakowski, T. J. et al. Science 358, 1318-1323 (2017).

3. Jabaudon, D. Nature Commun. 8, 16042 (2017).

4. Rakic, P. Science 241, 170-176 (1988)

5. O'Leary, D. D. M. Trends Neurosci. 12, 400-406 (1989).

6. Puelles, L. \& Rubenstein, J. L. R. Trends Neurosci. 26, 469-476 (2003).

7. Johnson, M. B.\& Walsh, C. A. Curr. Opin. Neurobiol. 42, 9-16 (2017).

8. Wamsley, B. \& Fishell, G. Nature Rev. Neurosci. 18, 299-309 (2017).

9. Radick, G. Nature 533, 293 (2016).

10.Weldon, W. F. R. Biometrika 2, 44-55 (1902).

11.Mi, D. et al. Science http://dx.doi.org/10.1126/ science.aar6821 (2018)

This article was published online on 5 March 2018.

\section{A cellular passage to the root interior}

Water-conducting tissues inside plant roots are surrounded by impermeable cells. This protective barrier is punctured by 'passage cells', which are thought to regulate nutrient uptake. How these cells form has now been revealed. SEE LETTER P.529

\section{SEDEER EL-SHOWK \& ARI PEKKA MÄHÖNEN}

$\mathrm{P}$ lants need to take up water and nutrients through their roots, while keeping out pathogens and toxins. To achieve this, the roots' inner transport tissues are enclosed in a protective, impermeable barrier of endodermal cells, interspersed with 'openings' in the form of a specific type of endodermal cell called a passage cell, which is thought to serve as a cellular gatekeeper, controlling access to the root interior ${ }^{1-4}$. On page 529, Andersen et al. ${ }^{5}$ describe molecular mechanisms that control the formation of passage cells, and show how the numbers of such cells are regulated by nutrient availability. The findings offer insights into the formation of these key plant cells, and link this to the patterning processes that form the embryonic root, providing an intriguing demonstration of the continuity of developmental mechanisms.

As endodermal cells develop, the impermeable polymers lignin and suberin are deposited in the cell walls. The deposits build up as the cells mature, forming an impermeable barrier ${ }^{4}$ that isolates the adjacent inner tissues from the root's outer cell layers and the soil. Once this process is complete, it is thought that nutrients and water can reach the interior only by means of passage cells ${ }^{4}$. These cells contain lignin deposited in an arrangement called the Casparian strip in their cell walls, but lack suberin, and thus offer a permeable route for molecular transport ${ }^{4,5}$. The extent of 'suberization' of endodermal cells is regulated by the

levels of the hormones abscisic acid (ABA) and ethylene, and can be reversibly affected by both nutrient availability and stress ${ }^{6}$. The extent of suberization is thought to be a key factor in the rate of nutrient uptake ${ }^{6}$. Although the role and regulation of suberization in endodermal cells are understood, the molecular mechanisms controlling the development of passage cells have remained a mystery.

Passage cells have been observed in many plants species ${ }^{1-3}$, and are formed in a region of the root above the growing root tip. Andersen et al. used the plant

"Plants can dynamically adjust the number of passage cells according to nutrient status." Arabidopsis thaliana as their model system. To confirm that passage cells form in its roots, they tracked the expression of a gene required for suberin synthesis by monitoring a fluorescent marker protein. They identified suberindeficient passage cells distributed at seemingly random locations along the root's length. The cells were consistently positioned around the root's circumference near the developing xylem, one of two types of water- and nutrient-conducting tissue (Fig. 1).

To investigate the nature of the link between xylem and passage cells, the authors tested plants in which genetic mutations altered the pattern of xylem development. Two of the mutant plants ${ }^{7,8}$ had fewer passage cells than normal. These plants had defects in the genes
AHP6 or LOG4, which encode proteins that act respectively in the signalling and biosynthesis of the hormone cytokinin. Cytokinin affects the transport and signalling of the hormone auxin to regulate the development and patterning of water-conducting tissues ${ }^{8,9}$. Auxin, in turn, drives the expression of AHP6, which encodes a protein that inhibits cytokinin signalling in the developing xylem ${ }^{7,9}$. The authors found that AHP6 protein (fused to a fluorescent marker protein) diffused from the developing xylem into the adjacent endodermal cells, probably through cell-connecting nanostructures called plasmodesmata, and that this process was required for passage-cell formation.

The finding led Andersen and colleagues to suspect that a feedback loop between cytokinin and auxin signalling might be involved in passage-cell formation. They therefore analysed plants expressing marker proteins to monitor the levels of cytokinin signalling. They found that passage cells have low levels of cytokinin signalling, or lack it altogether, unlike their neighbouring suberized endodermal cells. Analysis of another marker protein showing the level of auxin signalling revealed an auxin response in all of the endodermal cells near the developing xylem, including passage cells. Seedlings grown with auxin had more passage cells than did plants that did not receive an auxin boost. Conversely, seedlings treated with cytokinin contained fewer passage cells than untreated seedlings. Opposing roles for auxin and cytokinin are a ubiquitous theme in plant development, and the presence of such a feedback loop links passage-cell formation to patterning processes acting in the transport tissues and elsewhere.

The authors investigated whether auxin and cytokinin might affect ABA-mediated suberin deposition. Plants that were engineered to express cytokinin-signalling inhibitors throughout the endodermis had low suberin deposition in all endodermal cells, and this outcome was unaffected by the addition of ABA. However, it is unclear why ABA-mediated suberin deposition requires cytokinin signalling. Plants were engineered to express auxin-signalling inhibitors throughout the 